\title{
COMPARATIVE ANALYSIS OF LASER POLARIMETRY METHODS OF POLYCRYSTALLINE FILMS OF CEREBROSPINAL FLUID FOR POST-MORTEM INTERVAL ESTIMATION

\author{
Marta Garazdiuk ${ }^{1}$, Viktor Bachynskiy ${ }^{2}$, Oleh Vanchuliak ${ }^{3}$, Oleksandr Garazdiuk $^{4}$, \\ Yuliya Sarkisova ${ }^{5}$
}

\begin{abstract}
Obtaining the most accurate information about an object during its evaluation is one of the most urgent tasks of current forensic medical examination. That is why the application of new technologies in forensic medical expertise becomes an integral part of the technological support of forensic examination institutions.

Purpose: To compare the effectiveness of cerebrospinal fluid polycrystalline films, microscopic images, two-dimensional Stokes-polarimetry, and autofluorescent polarimetry in post-mortem interval estimation over long- and short-term time intervals.

Material and methods. The object of the study is cerebrospinal fluid donated from 70 corpses (experimental group) and 20 living persons (control group). Methods used: two-dimensional Stokes-polarimetry, autofluorescent polarimetry.

Results: Quantitative characteristics of the cerebrospinal fluid's polycrystalline films statistical moments allows us to establish relations between them and the duration of the post-mortem interval at a significant time interval. However, this method does not consider the morphology of the film of the cerebrospinal fluid and its coordinate structure. In contrast to this method, fluorescence microscopy considers the biochemical composition of the biological fluid and carries information about the concentration of molecular complexes of proteins, NADH, flavins, porphyrins, etc. In the early post-mortem period, changes in the cerebrospinal fluid begin precisely with changes in the concentration of biochemical compounds, and crystalline changes are secondary, so it can be argued that this method is most effective for diagnosing the progression of the time in the first hours after death.

Conclusions: Fluorescent laser polarimetric methods allow accurate estimation of the postmortem interval during the first hours after death. While, the polarization methods is more effective during the long-term periods of time, but has less precision.
\end{abstract}

UDC Classification: 616.1, DOI: 10.12955/cbup.v7.1445

Keywords: post-mortem interval, stokes-polarimetry, autofluorescence polarimetry, cerebrospinal fluid

\section{Introduction}

Clarification of postmortem tissue changes can help characterize the process of biological degradation and improve investigations in forensic practice, especially in the estimation of postmortem interval (PMI). Accordingly, the most urgent task of a forensic medical examination at the present is to obtain the maximum possible and most accurate information about the object in its study (Morikawa et al., 2016). That is why the application of new technologies in forensic medical expertise becomes an integral part of the technological support of forensic medical offices. However, a large number of the current methods used in practice, despite providing just enough information, in some cases do not allow to reliably and accurately answer the questions posed by the expert. The above-mentioned stimulates the development of an interdisciplinary approach, due to the intensive development of scientific and technological progress, primarily at the intersection of biological body optics, forensic medicine and modern analytical approaches. The implementation of this approach led to the emergence of works which considers the possibility of using laser polarimetric (LP) research methods to determine several important expert issues, in particular, the estimation of time after death (Ushenko et a.1, 2012).

LP techniques are based on the results of instrumental measurement of biological tissues (BT) values. They are among the most objective methods to describe changes that occur in a biological object under the influence of a combination of environmental factors and changes in BT. The method of laser microscopy polarization allows us to obtain important information about the morphological structure and properties of BT and human organism fluids. So, in accordance with the optics of BT and fluids,

\footnotetext{
${ }^{1}$ Higher State Educational Establishment of Ukraine "Bukovinian State Medical University”, Department of Forensic Medicine and Medical Law, Chernivtsi, Ukraine, m.garazdiuk@gmail.com

${ }^{2}$ Higher State Educational Establishment of Ukraine "Bukovinian State Medical University", Department of Forensic Medicine and Medical Law, Chernivtsi, Ukraine, sudmed@bsmu.edu.ua

${ }^{3}$ Higher State Educational Establishment of Ukraine "Bukovinian State Medical University", Department of Forensic Medicine and Medical Law, Chernivtsi, Ukraine, wanchuliak@gmail.com ${ }^{4}$ Higher State Educational Establishment of Ukraine "Bukovinian State Medical University", Internal Medicine Department, Chernivtsi, Ukraine, olexandr.harazdiuk@gmail.com

${ }^{5}$ Higher State Educational Establishment of Ukraine "Bukovinian State Medical University", Department of Forensic Medicine and Medical Law, Chernivtsi, Ukraine, liquiritia.s@gmail.com
} 
they consist of two phases: amorphous and optically anisotropic (crystalline) (Ushenko et al., 2012). Crystalline structures include coaxial fibrils that form collagen, elastin, myosin organic fibers, which are surrounded by fluids. The clear order of fibrillar proteins is an important feature used during analysis of BT. It should be noted that polarization microscopy carries information about the degree of ordering and crystallization of the BT structure (Ushenko et al., 2016).

There are numerous methods of BT and human body fluids analysis, which allow us to establish and examine those pathological conditions that were a prerequisite for death or led to it, as well as identify the PMI. Recently, considerable attention has been paid to the study of Cerebrospinal fluid (CSF). CSF is relatively isolated and maintains a relatively stable biochemical composition when the level of biochemical compounds in the blood fluctuates (Moghtaderi et al., 2017). It is explained by the presence of the blood-brain barrier.

A wide range of parameters is analyzed during the implementation of LP techniques. Since optical radiation has a vector character, for its thorough description, when it is going through the BT, it is necessary to use a set of indicators of intensity (amplitude), phase, frequency (wavelength) and state of polarization. The latter is characterized by the orientation in time of the electromagnetic-radiation vector. If this orientation in the plane of the wave front (for a plane wave) stays stationary during the measurement, the radiation is polarized, otherwise the light will be partially or completely depolarized after passage through the medium. The state of the polarization of light undergoes changes in radiation through the sample of the BT, as well as being reflected from its surface.

The purpose of the work. To compare the effectiveness of cerebrospinal fluid polycrystalline films, microscopic images two-dimensional Stokes-polarimetry, and autofluorescent polarimetry in postmortem interval estimation over long- and short-term time intervals.

\section{Materials and methods}

The objects of investigation are polycrystalline films (PF) of CSF, taken in 70 corpses of both sexes aged 29 to 85 years with accurately known times since death (the main experimental group) and 20 healthy volunteers (the comparison group). Time since death in the $1^{\text {st }}$ group ranges from 1 to 33 hours. Persons with suspected stroke or brain trauma have been excluded from investigation. CSF was taken and processed by a suboccipital puncture from the great occipital tank of a corpse and using lumbar puncture in healthy volunteers according to the Moghtaderi et al. (2017).

PF of CSF was formed in identical conditions by the placement a drop of CSF on optically homogeneous glass. The drying of films was performed at room temperature $\left(\mathrm{t}=22^{\circ} \mathrm{C}\right)$. Images of CSF PF were taken using a standard Stokes-polarimeter (Ushenko et al., 2012). Mathematical processing and analysis of the CSF PF images to establish the time-based dynamics of CSF postmortem changes was performed.

Coordinate distributions of the azimuth values and the ellipticity of polarization of SCF PF microscopic images were measured using the following methods (Ushenko et al., 2012):

1. Two-dimensional Stokes-polarimetry in different spectral bands of optical radiation - estimation of coordinate distributions of azimuth values and ellipticity of CSF PF ellipticity (see Fig. 1).

2. Fluorescent Stokes-polarimetry for autofluorescent biological layers study (see Fig. 2).

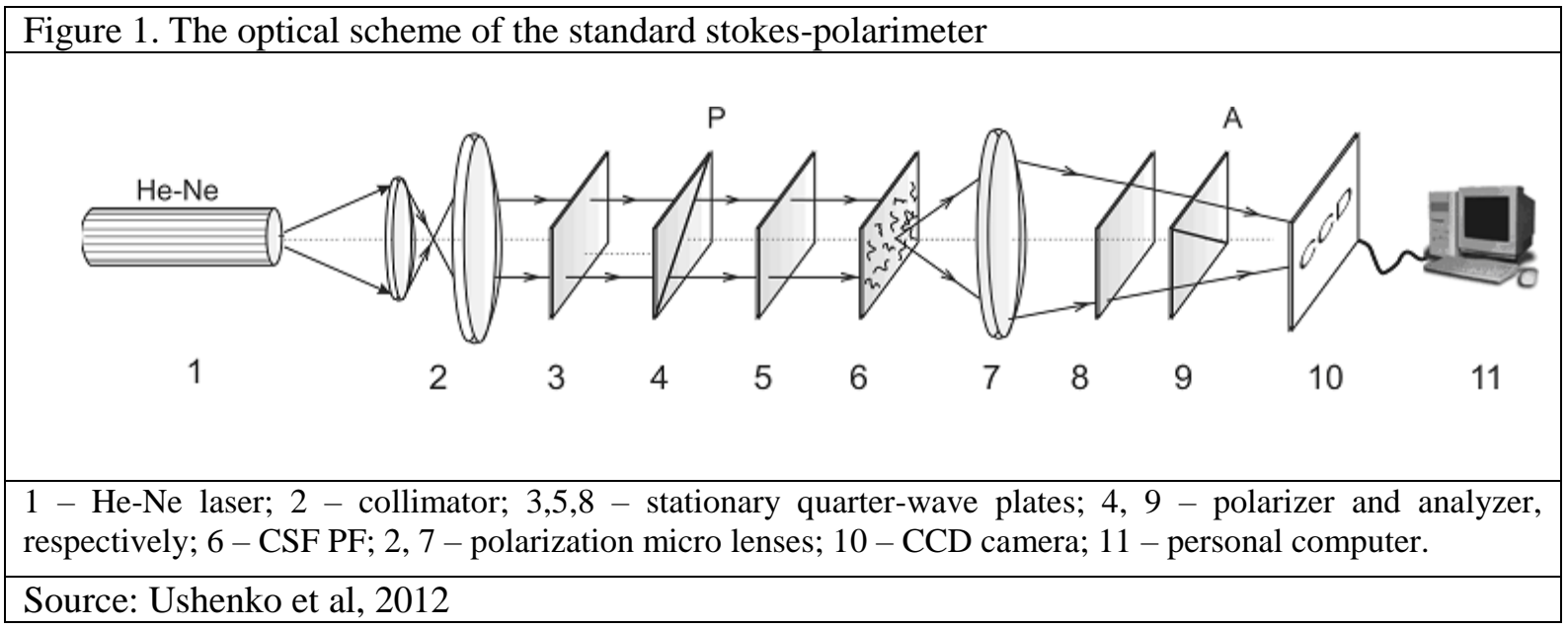




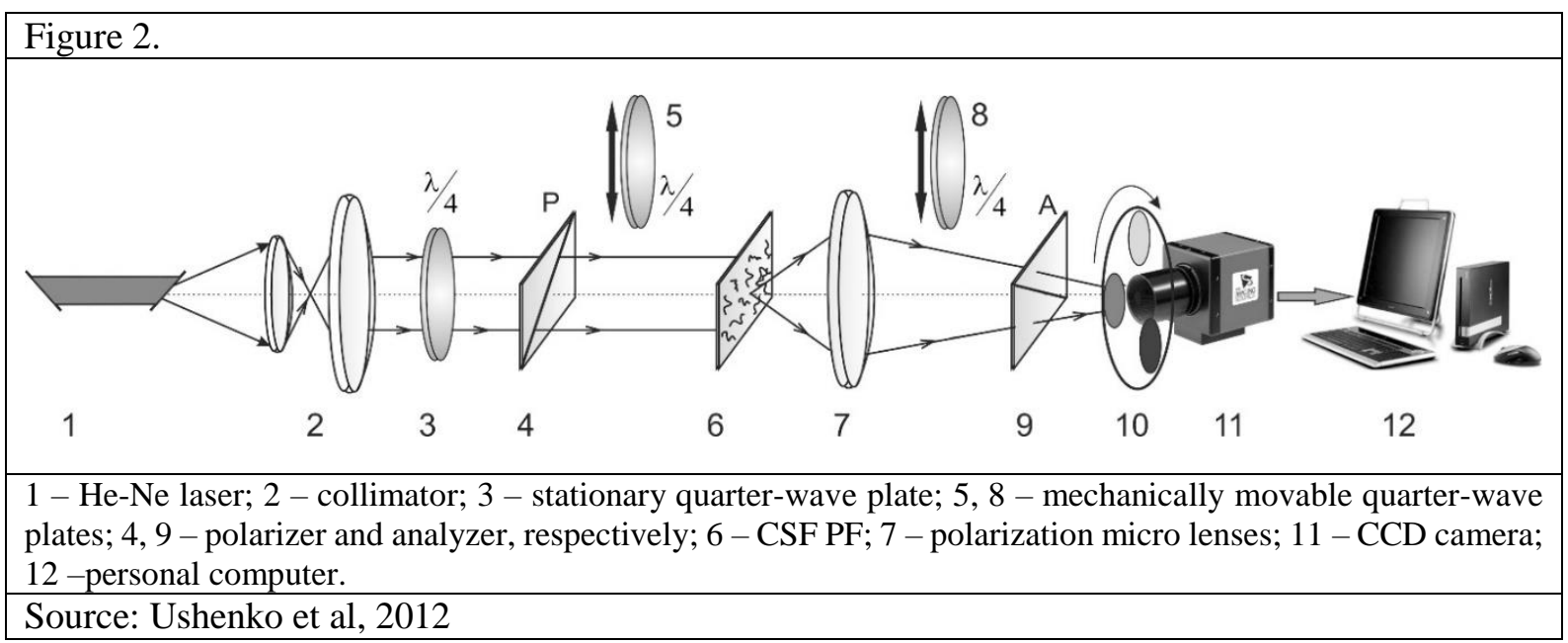

The description of the laser polarimeter and its modifications, as well as the methods for measuring the coordinate distributions of polarization parameters and the algorithm for postmortem interval estimation are detailed in scientific papers (Ushenko A. et al., 2012). The mean (statistical moment (SM) of the first order $\left(Z_{1}\right)$ ), dispersion (SM of the second order $\left(Z_{2}\right)$ ), asymmetry $\left(Z_{3}\right)$ and sharpness of the peak (kurtosis) of coordinate distributions $\left(\mathrm{Z}_{4}\right)$ of polarization and fluorescence image maps have been calculated for each CSF PF image.

\section{Results and conclusions}

SM of the 1-4 orders that characterize the distribution of azimuth and the ellipticity values of the CSF PF microscopic images polarization were different from zero and changed with a certain characteristic for each SM separately, depending on the time after the sampling of the CSF.

Analysis of two-dimensional mapping of azimuth and ellipticity (Table 1) of the CSF PF microscopic image polarization demonstrated the most significant changes in SM of the 3rd and 4th orders. However, for the ellipticity, the range of their change is less than for azimuth: the asymmetry increases by 2.12 times, the excess - by 2.42 times, while for the azimuth SM of 3rd order increased by 3.09 times, and the 4 th - by 2.83 times, respectively.

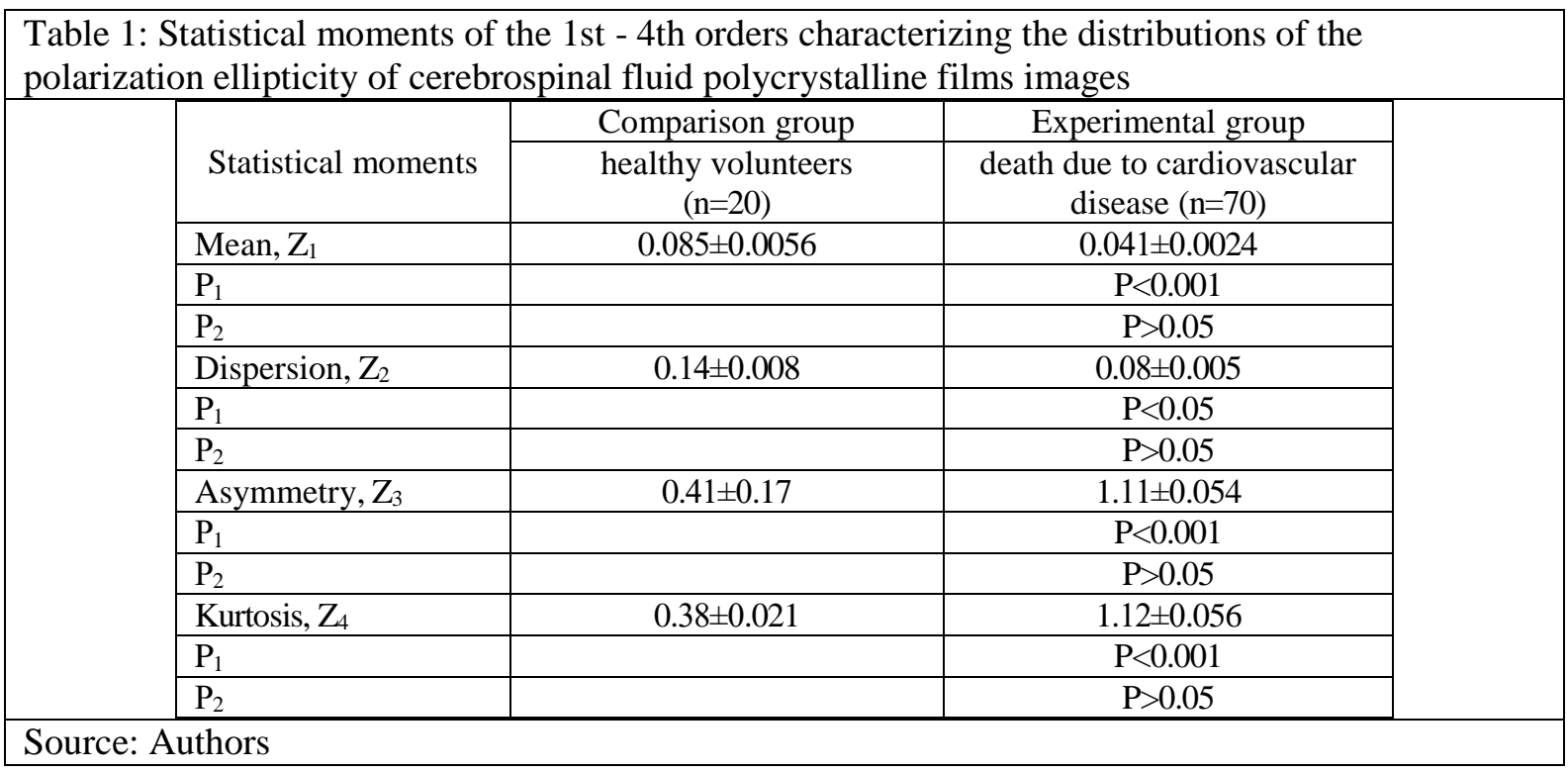

The comparative analysis of the histograms of the distribution of random values of the ellipticity of polarization demonstrates that the range of scattering decreases significantly more slowly than in the case of direct mapping of azimuth polarization - 1.7 times in 6 hours after death.

As a result, for the method of polarimetric mapping of azimuth and the ellipticity distributions of the polarization of CSF PF images in the time monitoring of posthumous changes in optical manifestations 
of polycrystalline networks, an interval of diagnostics of the PMI of 26-32 $\mathrm{h}$ with an accuracy of 45-60 minutes was established.

To compare the accuracy of the PMI estimation, the method of autofluorescent laser polarimetry was chosen. This is because when analyzing the image of a biological sample in the framework of statistical analysis using the Stokes-polarimetry, quantitative characteristics about the magnitude of SM can be obtained. The difference between polarization and autofluorescence images is that the polarization image carries information on the coordinate distribution of the polycrystalline structure of the PF of CSF in the form of azimuth and ellipticity distributions polarization, which are determined by the direction of the crystal and the concentration of its molecular structure, and the autofluorescent image gives information about the intensity of the re-radiation of molecular complexes PF of CSF with its irradiation by a laser. On this basis, the concentration of molecular complexes of proteins, NADH, flavin, porphyrins, etc. are judged (Garazdiuk et al., 2018).

Using the two-dimensional mapping method for the distribution of intensity of laser-induced fluorescence in the PF of CSF, its effectiveness was established in the time monitoring of posthumous changes of various biochemical molecular complexes of endogenous fluorophores. For more accurate PMI estimation the study was conducted in different spectral bands of optical radiation.

For the short-wave fluorescence range of protein molecules and NADH, an interval of 24 hours is established and the accuracy of the PMI estimation is $\pm 12.5 \mathrm{~min}$. For the medium-wave fluorescence range of the flavin and folic acid molecules, an interval of $28 \mathrm{~h}$ is established, and the accuracy of the PMI estimation is $17.5 \mathrm{~min}$. For the long-wavelength range of fluorescence of porphyrin molecules, an interval of $14 \mathrm{~h}$ was established and the accuracy of the PMI estimation was $7.5 \mathrm{~min}$.

Analysis of the parameters (interval and accuracy) for PMI estimation by the method of spectralselective two-dimensional mapping of the intensity distribution of the own fluorescence of various human biochemical molecular complexes PF of CSF of a human body revealed a significantly higher accuracy of the PMI estimation compared to Stokes-polarimetry at short-term time intervals (see Fig.3)

It is Figure 3. Time dependences of the statistical moments of the 1st and 3rd orders, which characterize the distribution of the fluorescence intensity values of the polycrystalline films of the cerebrospinal fluid in the "red" region of the spectrum

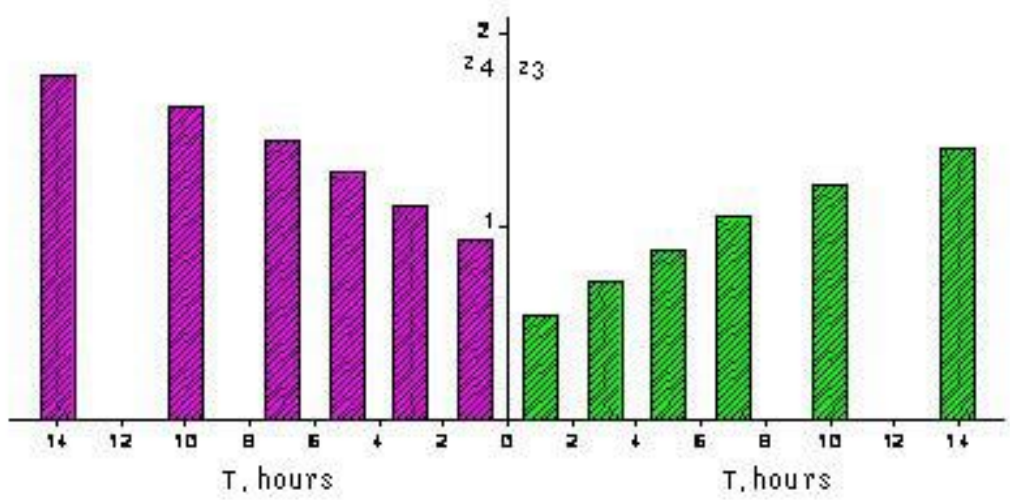

Source: Authors

\section{Conclusions}

A completely new methodical approach is proposed to solve the actual scientific and practically significant problem of developing a system of new forensic medical criteria for the estimation of the postmortem interval on the basis of studying films of cerebrospinal fluid.

Advantages of using a method for the two-dimensional mapping of the intensity distribution of the laserinduced fluorescence of cerebrospinal fluids on short-term time intervals compared to the Stokespolarimetry were found. This is because the post-mortem period changes in the cerebrospinal fluid begin with changes in the concentration of biochemical compounds, and crystalline changes are secondary. Therefore, fluorescence methods are precise on short-term time intervals for the determination of the 
postmortem interval while polarization allows for better estimation of this parameter on long-term time intervals, but with less precision.

\section{References}

Garazdiuk, M., Bachinskyi, V., Vanchuliak, O., Garazdiuk, O., \& Nechytailo, O. (2018). Time since death estimation by using the method of polycrystalline cerebrospinal fluid films images mutual polarization degree levels distributions twodimensional mapping with fine-scale spatial frequency filtration. Georgian medical news, (283), 166-170.

Moghtaderi, A., Alavi-Naini, R., \& Sanatinia, S. (2012). Lumbar puncture: techniques, complications and CSF analyses. In Emergency Medicine-An International Perspective. IntechOpen. Available from: http://cdn.intechopen.com/pdfs/31941/intechlumbar_puncture_techniques_complications_and_csf_analyses.pdf

Morikawa, K., Hyodoh, H., Matoba, K., Mizuo, K., Okazaki, S., \& Watanabe, S. (2016). Time-related change evaluation of the cerebrospinal fluid using postmortem CT. Legal medicine, 22, 30-35.

Ushenko, Y. A., Dubolazov, A. V., Angelsky, A. P., Sidor, M. I., Bodnar, G. B., Koval, G., ... \& Junisbekov, M. S. (2013, January). Laser polarization fluorescence of the networks of optically anisotropic biological crystals. In Optical Fibers and Their Applications 2012 (Vol. 8698, p. 869809). International Society for Optics and Photonics.

Ushenko, A. G., Dubolazov, A. V., Ushenko, V. A., \& Novakovskaya, O. Y. (2016). Statistical analysis of polarizationinhomogeneous Fourier spectra of laser radiation scattered by human skin in the tasks of differentiation of benign and malignant formations. Journal of Biomedical Optics, 21(7), 071110. 